Chirurgia (2017) 112: 486-492

No. 4, July - August

Copyright@ Celsius

http://dx.doi.org/10.21614/chirurgia.112.4.486

\title{
Breast Cancer Surgery in Images
}

\section{Alexandru Blidaru' ${ }^{1,2}$, Cristian Ioan Bordea ${ }^{1,2}$, Elena Ichim² ${ }^{2}$, Bassam El Houcheimi², Iulia Matei Purge ${ }^{1,2}$, Aniela Noditi ${ }^{1,2}$, Ionut Sterie ${ }^{2}$, Mirela Gherghe ${ }^{2}$, Mihaela Radu ${ }^{1,2}$}

"University of Medicine and Pharmacy "Carol Davila", Bucharest, Romania

2Department of Surgical Oncology, "Prof. Dr. Al.Trestioreanu" Institute of Oncology, Bucharest, Romania

Corresponding author:

Cristian loan Bordea, MD

University of Medicine and Pharmacy

"Carol Davila" , Bucharest, Romania

Department of Surgical Oncology,

"Prof. Dr. Al. Trestioreanu" Institute of

Oncology, Bucharest, Romania

E-mail: drbordea@yahoo.com
As a picture speaks more than a thousand words, we tried to reflect, at least in part, on our surgical day-to-day practice on the 2nd Oncological Surgery Department of "Prof. Dr. Al. Trestioreanu" Bucharest Institute of Oncology in the field of breast cancer. And how could we not talk about imaging in breast cancer, when one of the reasons for low breast cancer mortality at present is early detection, which is performed through imaging investigations.

Mammographic screening is recognized as a success in this area (Fig. 1), but moreover, mammary ultrasound, a newer addition to this domain, also allows the detection of infraclinical cancers (Fig. 2).

The localization of these infraclinical lesions can be obtained preoperatively by mammographic technique using a harpoon (Fig. 3).

Under ultrasound guidance we can not only puncture cysts (Fig. 4), but also perform mammary biopsies for suspicious lesions (Fig 5).

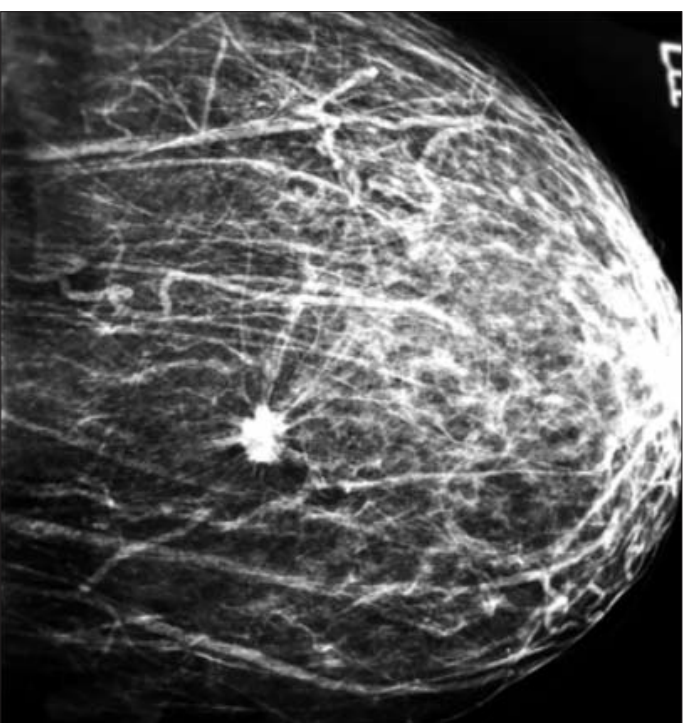

Figure 1. Mammography - BIRADS 5 lesion 


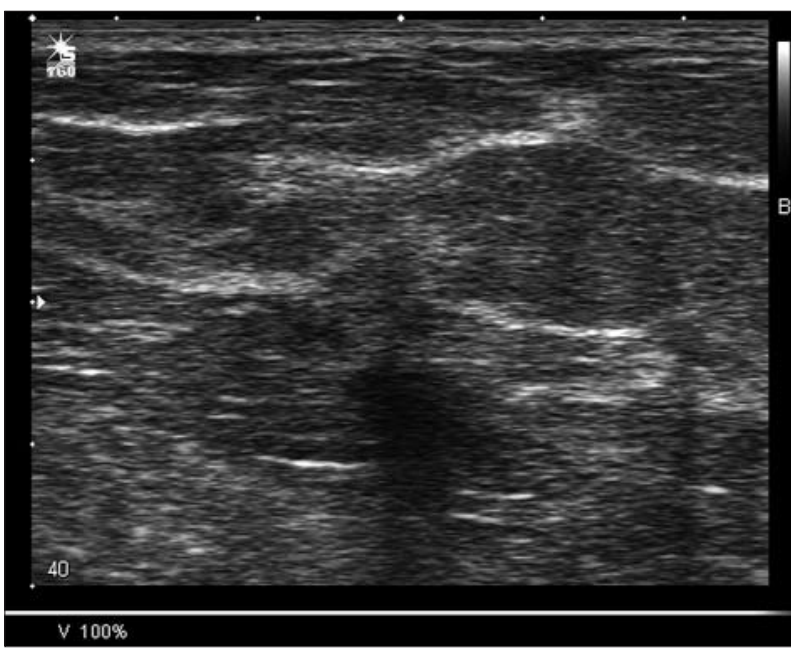

Figure 2. Breast ultrasound - BIRADS 5 lesion

Early diagnosis allows in most cases conservative treatment because of the small size of the tumour (Fig. 6), with a very good aesthetic result (Fig. 7).

Selective axillary dissection and avoidance of unnecessary complete axillary dissection are achieved through biopsy of the sentinel lymph node. At "Prof. Dr. Al. Trestioreanu" Bucharest Institute of Oncology we implemented the technique of identifying the sentinel lymph node using a radioactive tracer in 2006 and we have carried out so far over one thousand such procedures (Figs. 8, 9, 10).

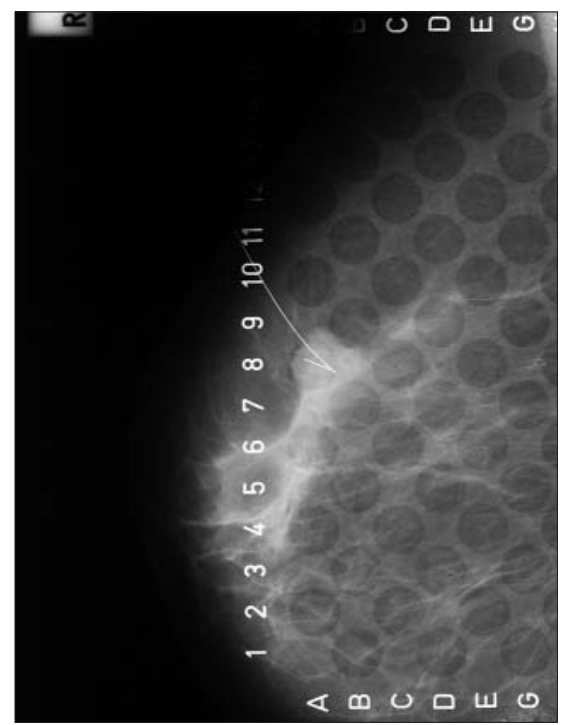

Figure 3. Hook-wire localization for non-palpable breast lesion
Sometimes oncoplastic surgery allows not only for breast preservation, but also safer surgery with better aesthetic result (Fig. 11).

Due to the extent of the disease, there are situations where mastectomy is mandatory (Fig. 12).

The changes in body image after mastectomy are important. Immediate breast reconstruction or at a distance attempts to solve this aesthetic problem and reduce the psychological impact. Since 1999 we have been performing immediate reconstruction and at a distance postmastectomy using implants at "Prof. Dr. Al. Trestioreanu" Bucharest Institute of Oncology (Fig. 13).

We perform various types of mastectomy depending on the stage of the disease, the characteristics of the patients, and the oncological treatment plan. (Figs. 14, 15, 16, 17)

The association of synthetic meshes allows for better aesthetic results (Figs. 18, 19, 20, 21)

Video-assisted surgery also finds its place in this pathology, easing dissection (Fig. 22).

We have performed both immediate postmastectomy reconstructions with or without preservation of the areola and nipple, as well as at a distance (Fig.s 23, 24, 25).

For patients with unilateral breast cancer and mutations of BRCA 1 or 2 genes, we performed bilateral mastectomy with immediate reconstruction (Figs. 26, 27).

This images shows how much breast cancer surgery has changed.

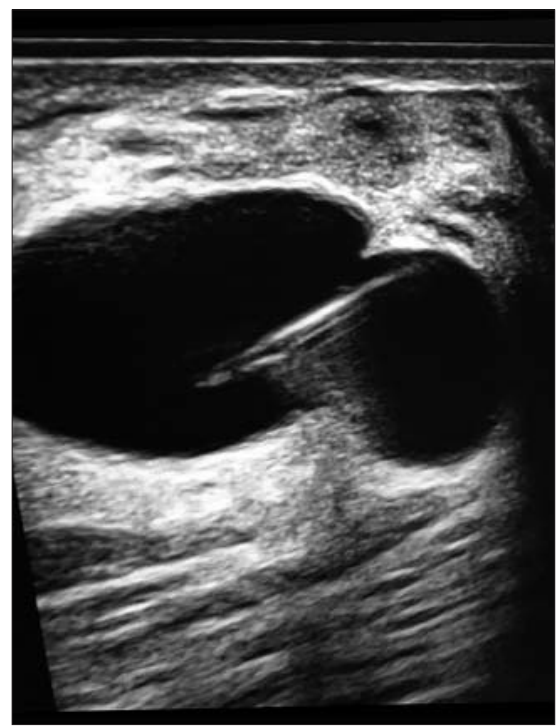

Figure 4. Breast cyst - FNAc under ultrasound guidance 


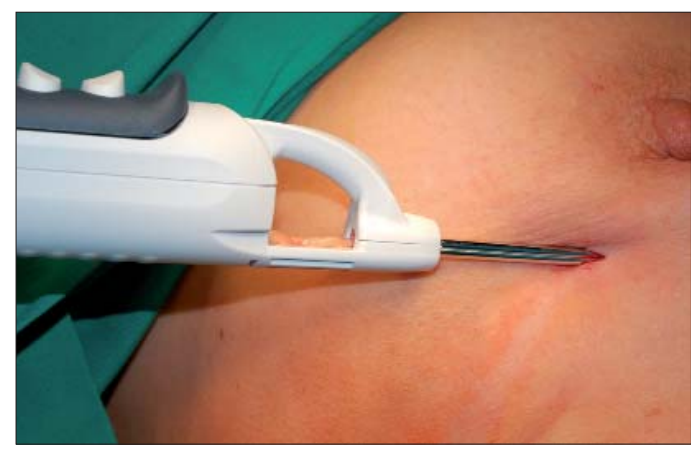

Figure 5. Needle core biopsy using Mammotome

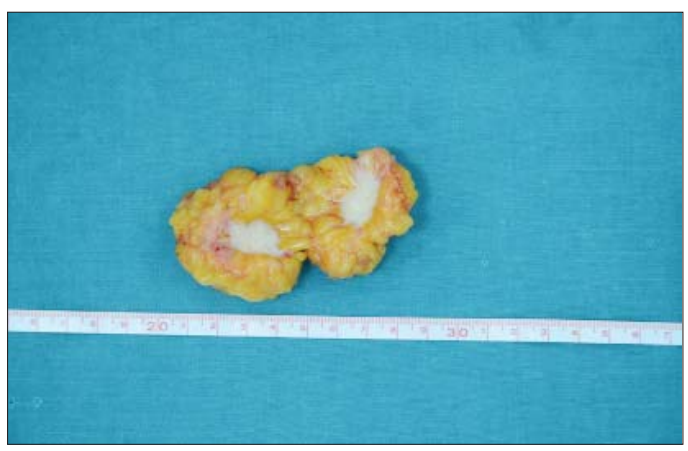

Figure 6. Macroscopic aspect of breast cancer
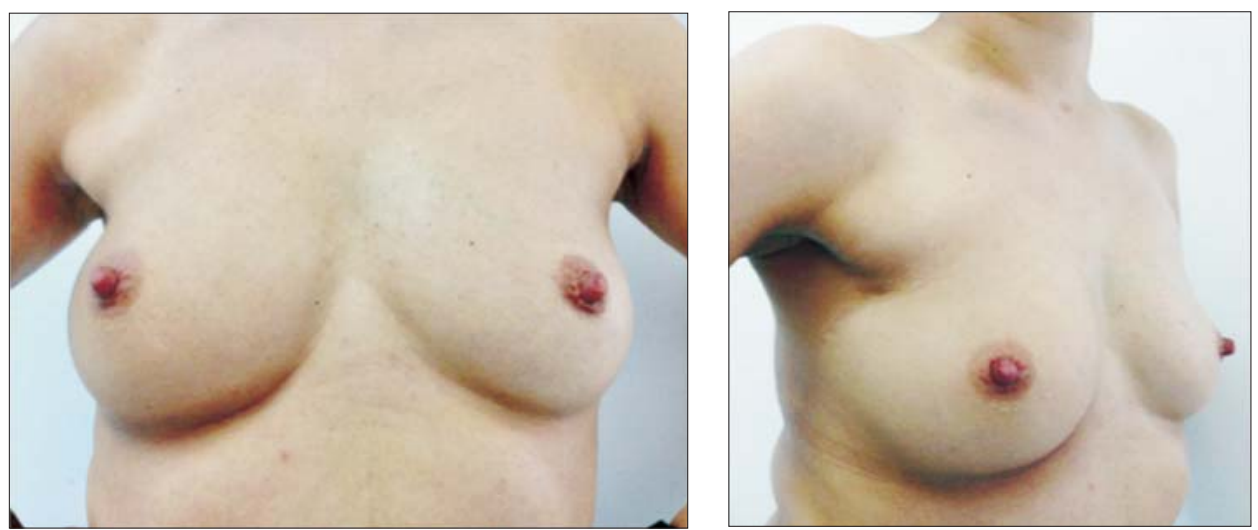

Figure 7. Breast conservative treatment for right breast cancer
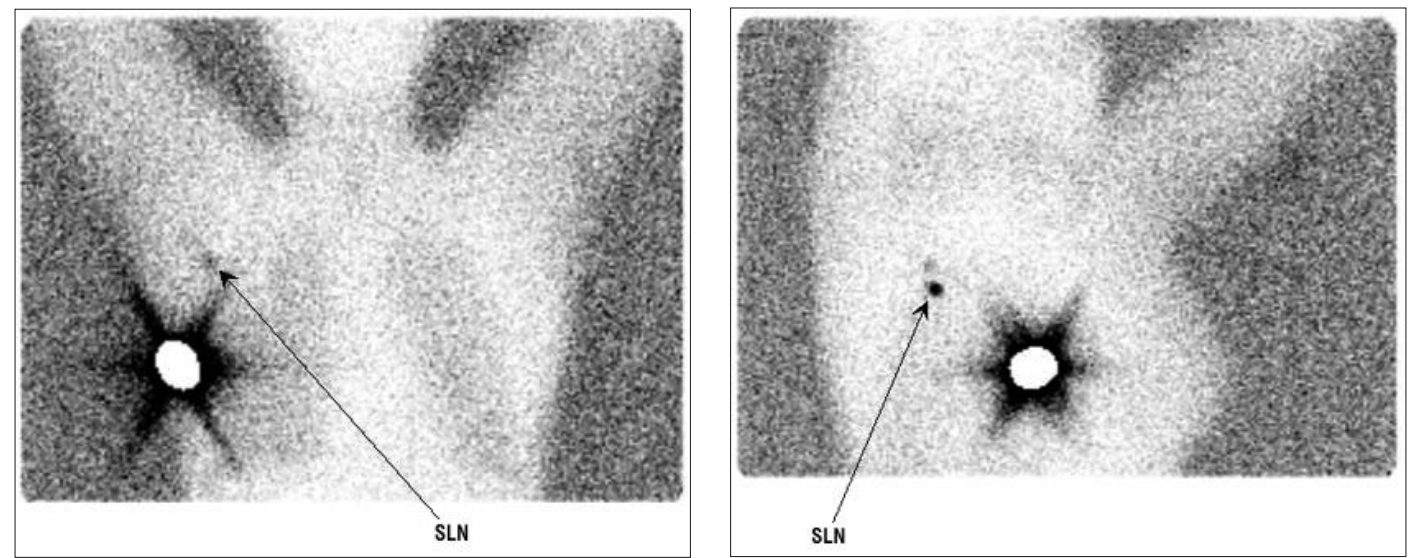

Figure 8. Preoperative lymphoscintigraphy for sentinel lymph node detection using radioactive tracer 


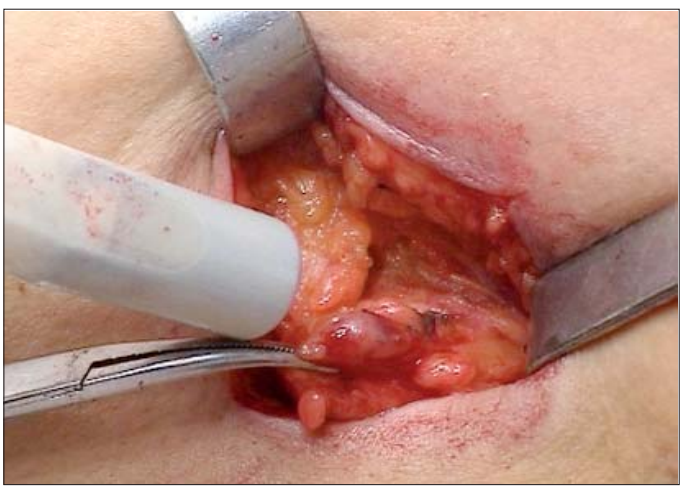

Figure 9. Sentinel lymph node biopsy - intraoperatory aspect

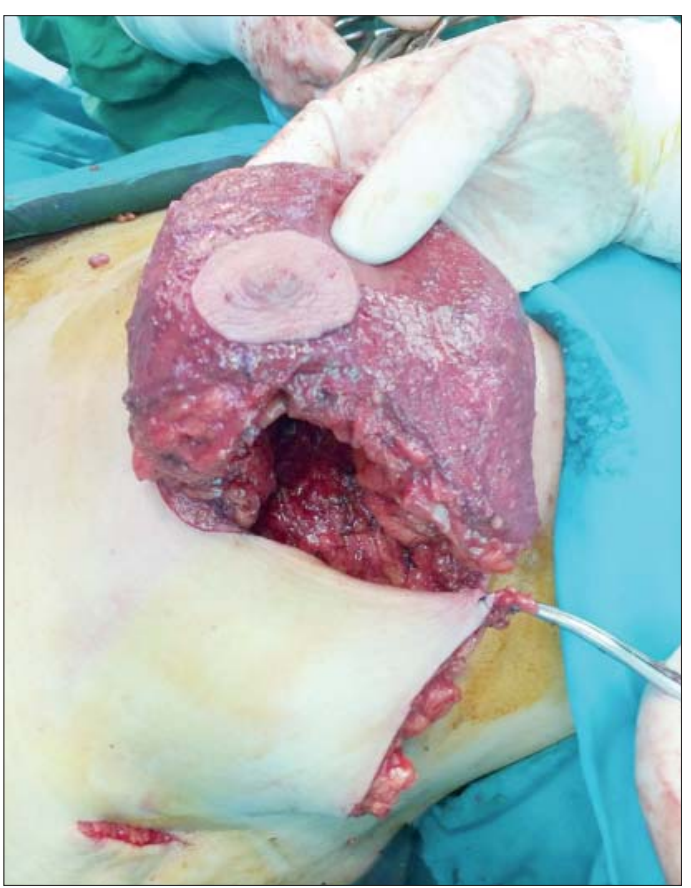

Figure 11. Therapeutic reduction mammoplasty for breast cancer

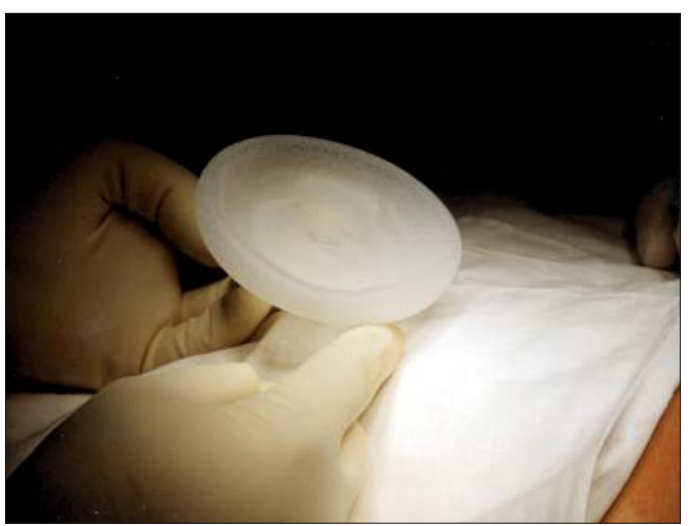

Figure 13. Saline filled implant for breastreconstruction

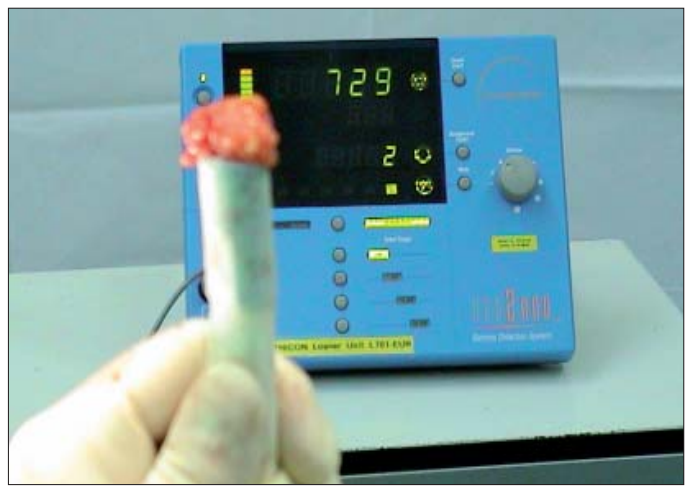

Figure 10. Sentinel lymph node identified using radioactive tracer

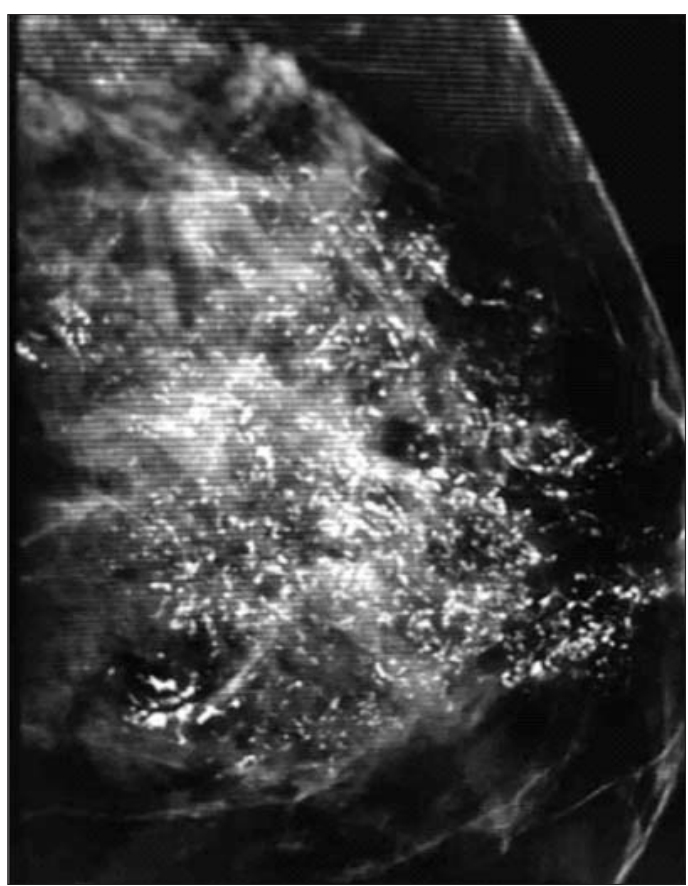

Figure 12. Multiple microcalcifications on mammography - DCIS - surgical indication: mastectomy

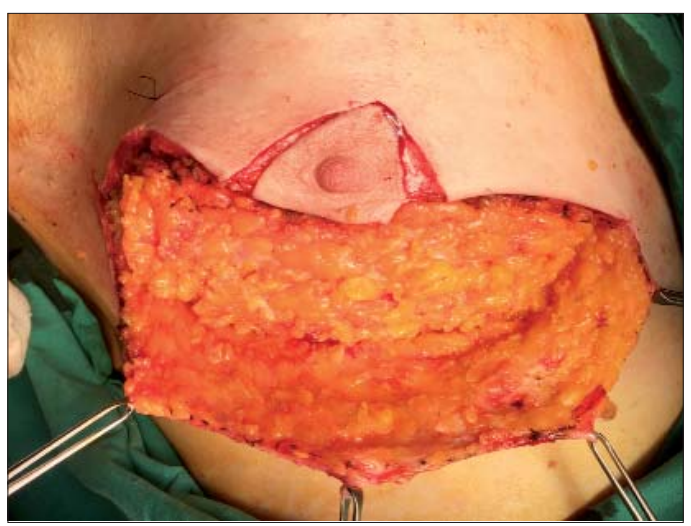

Figure 14. Goldilock mastectomy 

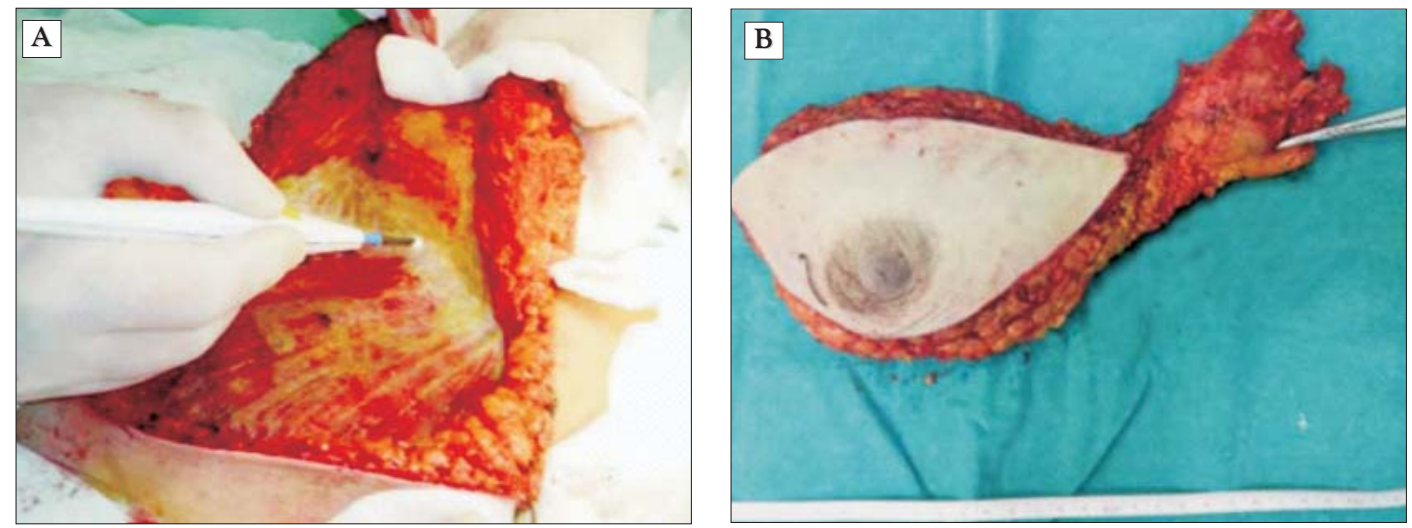

Figure 15. (A) Mastectomy - pectoralis fascia layer. (B) Mastectomy specimen - biopsy place marked with stitch

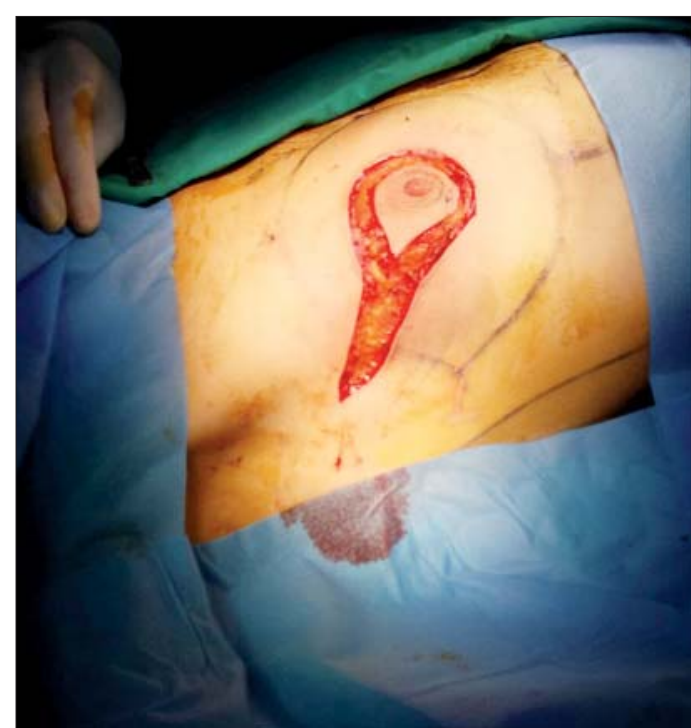

Figure 16. Skin sparing mastectomy incision

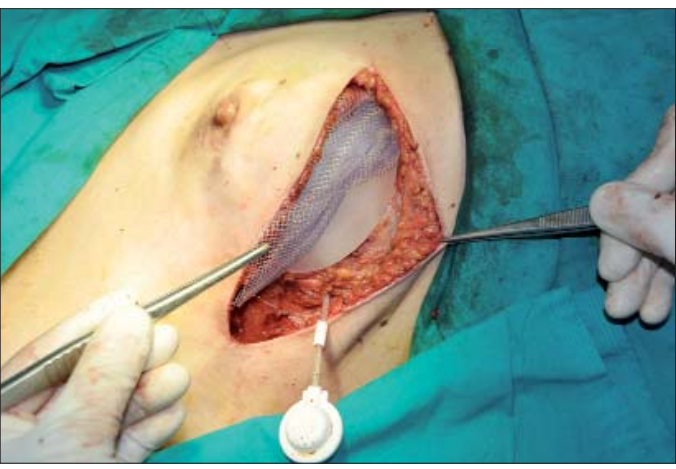

Figure 18. Immediate breast reconstruction after mastectomy with implant and mesh

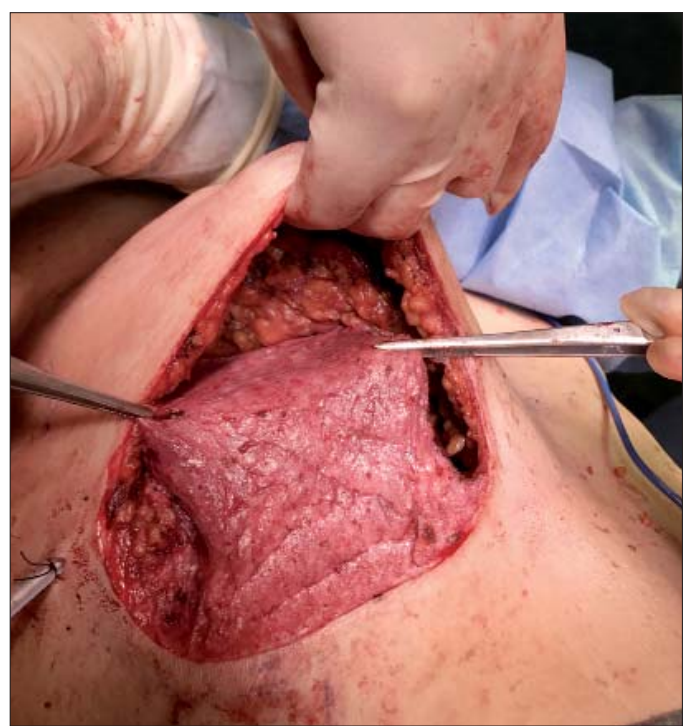

Figure 17. Mastectomy with deepithelialised skin flap for implant covering

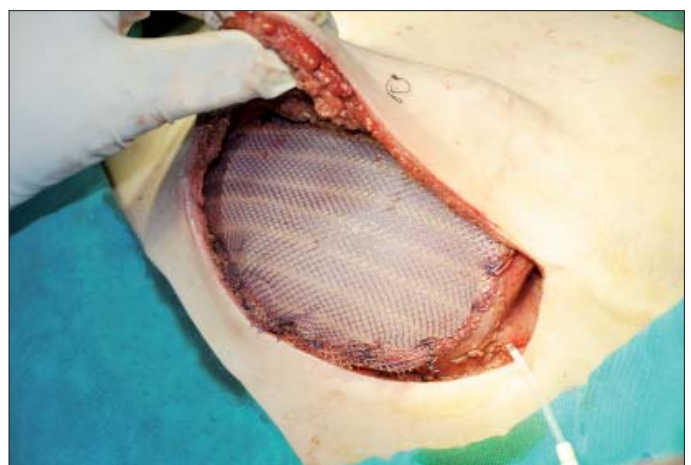

Figure 19. Immediate breast reconstruction with Becker implant and mesh 


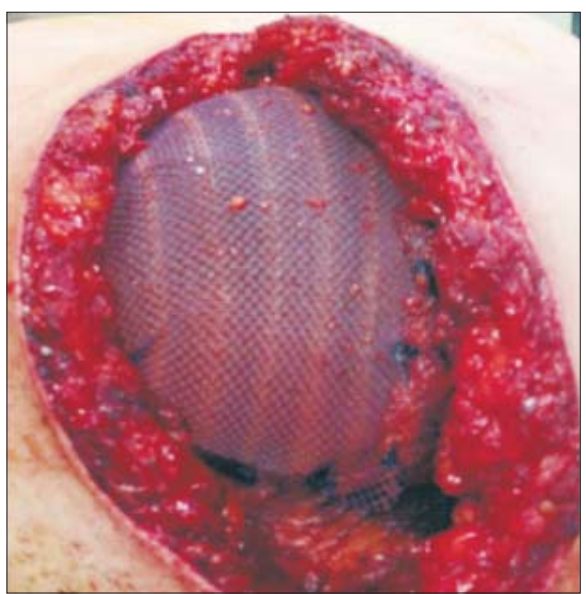

Figure 20. Mesh covering breast implant

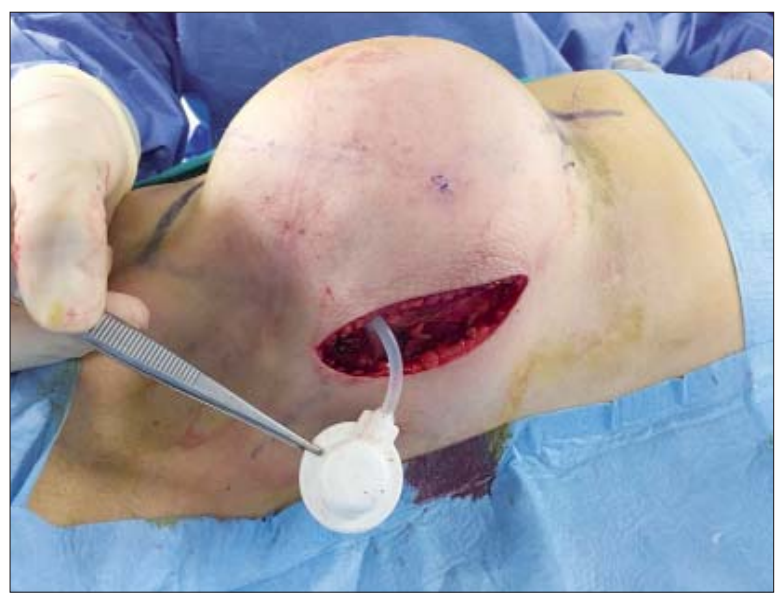

Figure 21. Delayed breast reconstruction with tissue expander

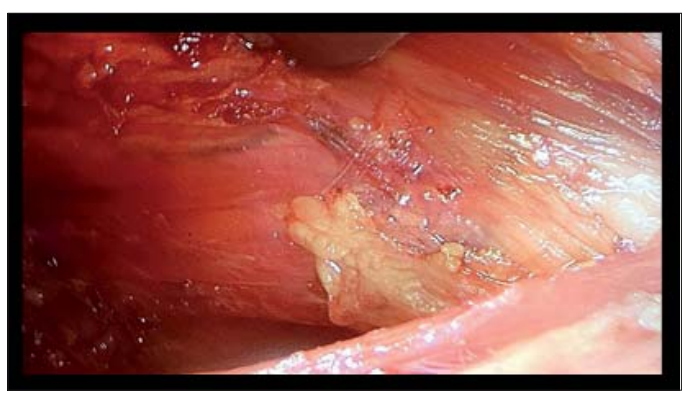

Figure 22. Video assisted breast reconstruction with retropectoral implant placement

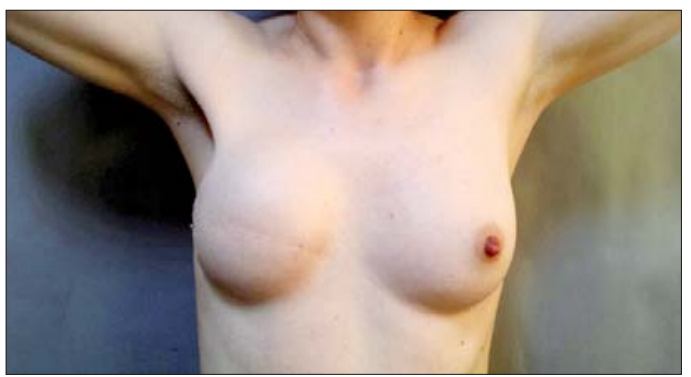

Figure 24. Skin sparring mastectomy and immediate breast reconstruction using implant

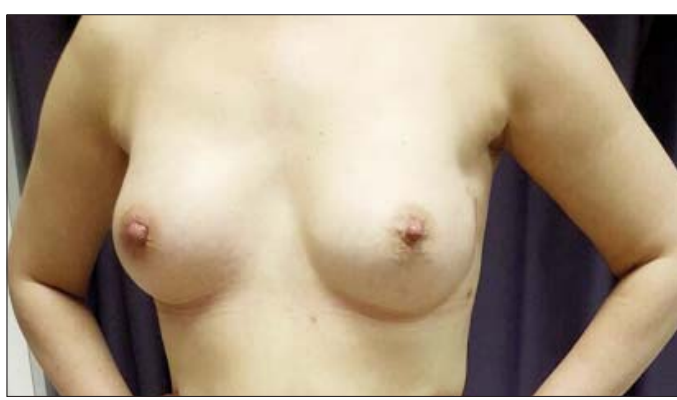

Figure 25. Nipple sparring mastectomy and immediate breast reconstruction using implant
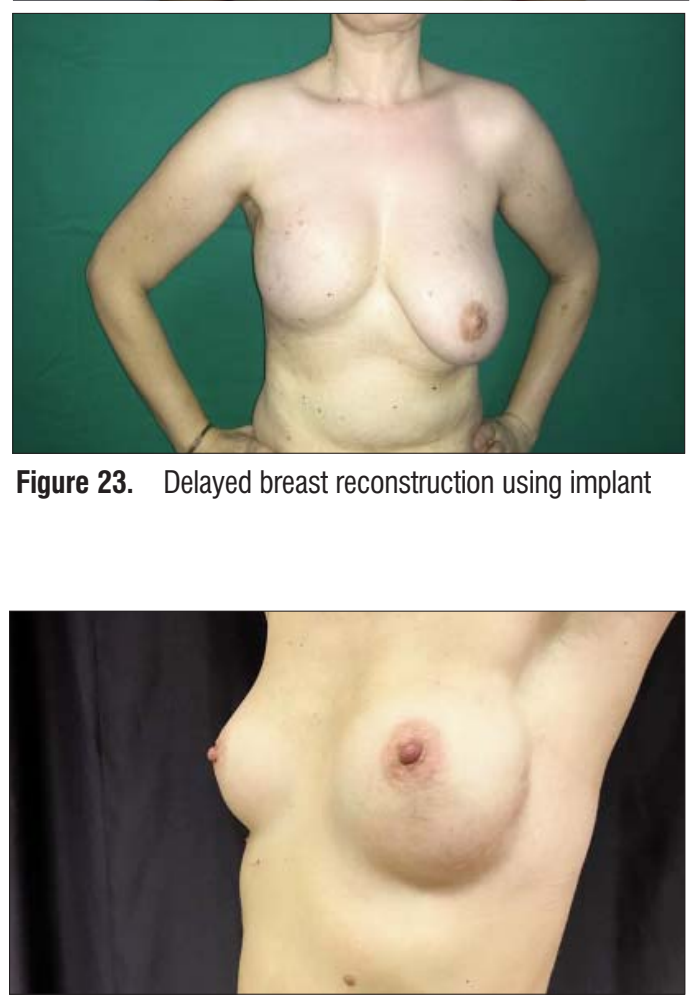

Figure 23. Delayed breast reconstruction using implant

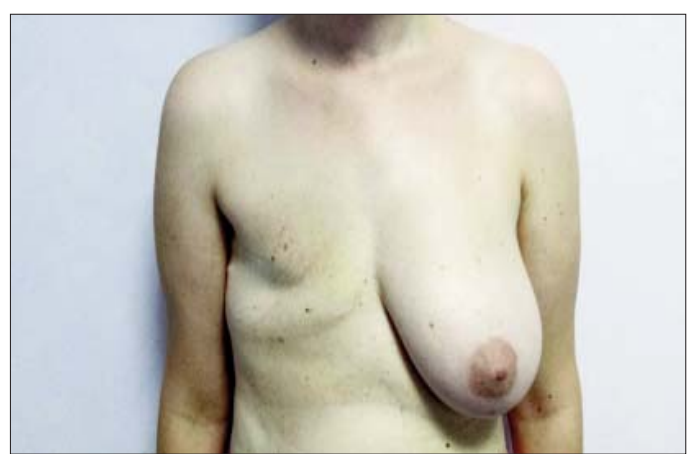



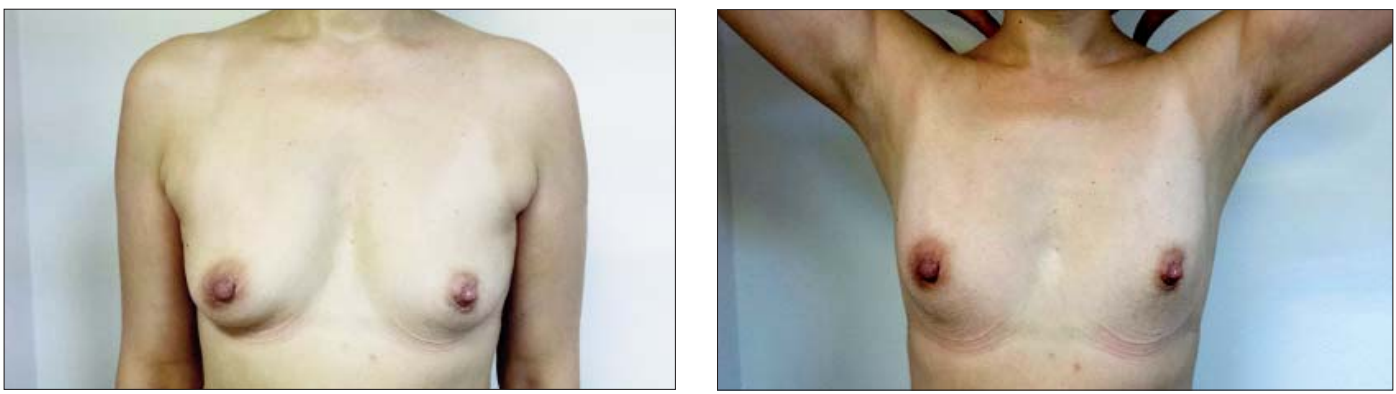

Figure 26. Right breast cancer and BRCA 1 mutation - preoperatory aspect
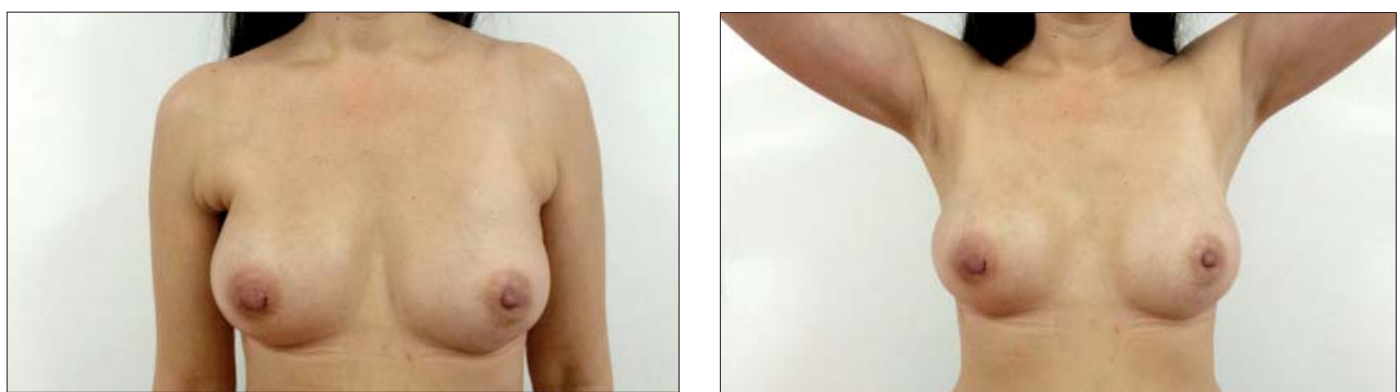

Figure 27. Bilateral nipple sparring mastectomy, right axillary lymph node dissection and immediate breast reconstruction with implants - post operatory aspect 